\title{
Role of Turnover in Active Stress Generation in a Filament Network
}

\author{
Tetsuya Hiraiwa ${ }^{1,2,3}$ and Guillaume Salbreux ${ }^{1,4}$ \\ ${ }^{1}$ Max Planck Institute for the Physics of Complex Systems, Nöthnitzer Straße 38, 01187 Dresden, Germany \\ ${ }^{2}$ Fachbereich Physik, Freie Universität Berlin, Berlin 14195, Germany \\ ${ }^{3}$ Department of Physics, Graduate School of Science, The University of Tokyo, Tokyo 113-0033, Japan \\ ${ }^{4}$ The Francis Crick Institute, 44 Lincoln's Inn Fields, London WC2A 3LY, United Kingdom
}

(Received 22 July 2015; published 6 May 2016)

\begin{abstract}
We study the effect of turnover of cross-linkers, motors, and filaments on the generation of a contractile stress in a network of filaments connected by passive cross-linkers and subjected to the forces exerted by molecular motors. We perform numerical simulations where filaments are treated as rigid rods and molecular motors move fast compared to the time scale of an exchange of cross-linkers. We show that molecular motors create a contractile stress above a critical number of cross-linkers. When passive crosslinkers are allowed to turn over, the stress exerted by the network vanishes due to the formation of clusters. When both filaments and passive cross-linkers turn over, clustering is prevented and the network reaches a dynamic contractile steady state. A maximum stress is reached for an optimum ratio of the filament and cross-linker turnover rates. Taken together, our work reveals conditions for stress generation by molecular motors in a fluid isotropic network of rearranging filaments.
\end{abstract}

DOI: 10.1103/PhysRevLett.116.188101

The cell cortical cytoskeleton is essential in processes involving cell shape changes [1,2]. In the cortex, myosin molecular motors are assembled in a bipolar filamentous structure which binds to actin filaments and generates forces by consuming the chemical energy of the hydrolysis of adenosine triphosphate. The action of myosin motors result in the generation of an active, contractile stress whose spatial distribution in the cortex plays a key role in cellular morphogenetic processes [3-5].

In living cells, passive and active cross-linkers and actin filaments are continuously exchanged between the cortex and the cytosol [1]. As a result, cytoskeletal networks can release elastic stresses stored in the network and undergo large-scale flows. Significant progress has been achieved to understand stress generation in networks with permanent filaments and fixed or unbinding cross-linkers, through in vitro studies and a theoretical analysis of actomyosin networks [6-15]. It is unclear, however, what is the role of turnover in stress generation and how filament networks can simultaneously rearrange and exert a permanent internal active stress. In vivo experiments suggest that the rate of turnover is a major determinant of force generation by actomyosin networks $[16,17]$.

We ask here how the rate of turnover of passive crosslinkers and actin filaments influence the active stress generated by motors in the network. We study a simplified mechanical model for a cytoskeletal network in two dimensions whose constituents are turning over [Fig. 1(a)]. Filaments and motors are treated as rigid rods. Filaments have a polarity represented by arrows in Fig. 1. Passive cross-linkers are assumed to be pointlike and constrain the position of the filaments on which they are attached. Filaments are able to rotate freely around the cross-linker position and around motor heads. Motor heads exert an active force $\boldsymbol{f}_{m}$ with a constant magnitude $\left|\boldsymbol{f}_{m}\right|=f_{0}$ on filaments, oriented toward the reverse direction of the arrow (the minus end of actin filaments).

To obtain forces acting on filaments, we introduce the effective mechanical potential, $U=W+\lambda$. $\boldsymbol{g}\left(\boldsymbol{x}_{f, i}, \boldsymbol{n}_{f, i}, \boldsymbol{x}_{m, k}, \boldsymbol{n}_{m, k}\right)$, where $W=-f_{0} \sum_{\langle k, i\rangle} s_{k i}$ is the work due to motor active forces, with $s_{k i}$ being the position

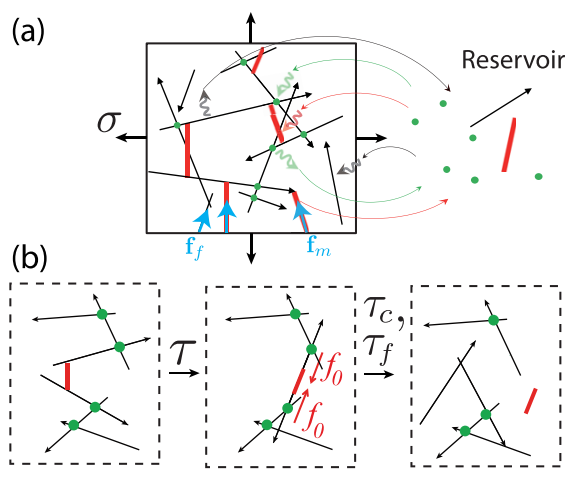

FIG. 1. (a) Schematic illustration of a 2D cytoskeletal network (left). Filaments are represented by black arrows, motors by red bars, and cross-linkers by green dots. The network exerts a stress $\sigma$ on the boundaries of the box, arising from tensions acting within the filaments $\left(\mathbf{f}_{f}\right)$ and within the motors $\left(\mathbf{f}_{m}\right)$. Motors move towards the arrowhead of filaments (for actin, the arrowhead corresponds to the barbed end). Network components stochastically exchange with a reservoir (right). (b) Motors move on filaments and rearrange the network on a time scale $\tau$. Crosslinkers and actin filaments turn over on longer time scales $\tau_{c}>\tau$ and $\tau_{f}>\tau$. 
of the $k$ th motor head on the $i$ th filament relative to the filament center of mass, and the sum $\sum_{\langle k, i\rangle}$ is performed for all of the pairs of filaments $(i)$ and motors $(k)$ connected with each other. In addition, geometrical constraints arise from the conditions that cross-linkers and motor heads are firmly attached to filaments, and that motor filaments have a fixed length. Lagrange multipliers $\lambda$ impose these constraints, $\boldsymbol{g}\left(\boldsymbol{x}_{f, i}, \boldsymbol{n}_{f, i}, \boldsymbol{x}_{m, k}, \boldsymbol{n}_{m, k}\right)=\mathbf{0}$, where $\boldsymbol{x}_{f, i}$ and $\boldsymbol{n}_{f, i}$ $\left(\boldsymbol{x}_{m, k}\right.$ and $\left.\boldsymbol{n}_{m, k}\right)$ indicate the center of mass position and orientation unit vector of the $i$ th filament ( $k$ th motor). The motion of motors is taken into account by writing that the position of attachment of the $k$ th motor $s_{k i}$ relative to the center of mass of the filament $i$ follows the dynamic equation

$$
\mu \frac{d s_{k i}}{d t}=-\frac{\partial U}{\partial s_{k i}},
$$

with $\mu$ being a scalar friction coefficient arising from translational friction between the motor heads and the filament [18-20]. Motors have a typical velocity $v_{m}=$ $f_{0} / \mu$, and we introduce a reference time scale $\tau=l_{f} \mu / 2 f_{0}$. We neglect viscous forces arising from the fluid around the network compared to motor-filament friction, and the position and orientation of filaments $\boldsymbol{x}_{f, i}, \boldsymbol{n}_{f, i}$ and motors $\boldsymbol{x}_{m, k}, \boldsymbol{n}_{m, k}$ are relaxed instantaneously.

To fix ideas, cortical actomyosin networks in a cell have a mesh size $\xi \sim 20-250 \mathrm{~nm}[21,22]$ and the typical cell diameter is several tens of micrometers. We therefore expect actin filaments to have a length $l_{f}$ of the order $0.1-1 \mu \mathrm{m}$, smaller than their persistence length $l_{p}=16 \mu \mathrm{m}$ [23]. Myosins move on actin filaments with velocity $v_{m} \sim 0.1-3 \mu \mathrm{m} / \mathrm{s}$. [24,25]. The characteristic time for myosins to move on a filament is $\tau \sim l_{f} / v_{m} \sim 0.03-10 \mathrm{~s}$. To compare this time scale to the effect of viscous stresses arising in the solvent of viscosity $\eta$, we note that the velocity of a filament in the network subjected to a force $f_{0}$ is $\sim f_{0} \ln \left(\xi / r_{f}\right) /\left(\eta l_{f}\right)$ from slender-body theory [26] taking into account the hydrodynamic screening effect [27], giving a time scale $\tau_{v} \sim l_{f}^{2} \eta /\left[f_{0} \ln \left(\xi / r_{f}\right)\right]$, with $r_{f} \simeq$ $5 \mathrm{~nm}$ being the radius of a filament. Taking the force exerted by a myosin $f_{0} \sim 6-12 \mathrm{pN}, \eta \simeq 10^{-3} \mathrm{~Pa} \cdot \mathrm{s}$ for water, $\tau_{v} \sim 10^{-4} \mathrm{~s} \ll \tau$. Finally, we expect the characteristic times $\tau_{c}$ and $\tau_{f}$ of the cross-linker and actin filament turnover to be of the order of $10 \mathrm{~s}-1 \mathrm{~min}$ [28,29], slow compared to these time scales, such that $\tau_{c}>\tau$ and $\tau_{f}>\tau$.

Configuration with one motor and two filaments.-We start by discussing the dynamics of configurations involving one motor attached to two filaments [Fig. 2(a)]. The two filaments can be connected to a cross-linker, itself connected to the external network, here considered to be fixed in space. Depending on the positions of the cross-linkers, the two filaments can be completely free [Fig. 2(b)(i)], cross-linked to each other [Fig. 2(b)(ii)], or to the external

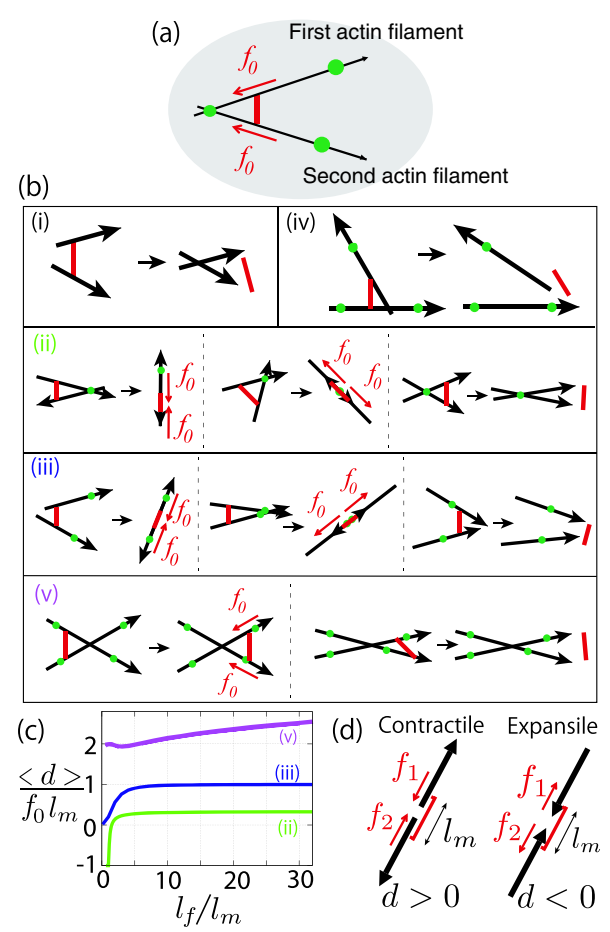

FIG. 2. Contractile and expansile configurations for one motor and two filaments. (a) Two filaments are attached by fixed crosslinkers to the external network. (b) Possible initial configurations for one motor, two filaments, and one or several cross-linkers, and final configurations. (c) Average strength of the contractile force dipole generated by the molecular motor $\langle d\rangle$ in the final configurations (ii),(iii),(v) where the motor does not detach, as a function of the filament length $l_{f}$. (d) Contractile (force dipole $d>0)$ and expansile $(d<0)$ configurations.

network [Fig. 2(b)(iii)-(v)]. By averaging over possible initial configurations [30], we evaluate whether motors form on average positive or negative force dipoles at steady state [Fig. 2(c)]. The motor force dipole is $d=\left(l_{m} / 2\right) \mathbf{n}_{12} \cdot\left(\mathbf{f}_{1}-\mathbf{f}_{2}\right)$, with $l_{m}$ being the length of the motor, $\mathbf{n}_{12}$ the unit vector giving the motor orientation, and $\mathbf{f}_{1}$ and $\mathbf{f}_{2}$ the forces exerted by the motor ends on the filaments [see Fig. 2(d) and the Supplemental Material [30]].

In cases shown in Figs. 2(b)(i) and 2(b)(iv), filaments are moved relative to each other until the motor detaches, so that a motor-induced force dipole acts on a transient time $\tau$, which can be neglected in the quasistatic limit $\tau \ll \tau_{c}$ [Fig. 2(b)]. In the cases shown in Figs. 2(b)(ii), 2(b)(iii), and 2(b)(v), filaments relax to a steady configuration where the motor exerts a constant force dipole, always positive on average in the cases shown in Figs. 2(c)(iii) and 2(c)(v). In the case shown in Fig. 2(c)(v), $\mathbf{f}_{1}$ and $\mathbf{f}_{2}$ include the forces exerted by the motor themselves and by geometrical constraints [30]. As shown in Ref. [9], the bias towards contractile states arises from instabilities of expansile configurations [30]. The average force dipole $\langle d\rangle$ vanishes for pointlike motors, $l_{m} \rightarrow 0$ [14]. 
Numerical simulations of networks with turnover.-We next numerically simulated a network of $N_{f}$ filaments, $N_{m}$ motor rods, and $N_{c}$ passive cross-linkers in a square box of width $W$ with periodic boundaries (Fig. 1). The frame of the box is not allowed to deform. The geometrical constraints $g$ are enforced by stiff linear springs. Initial conditions are obtained by randomly positioning filaments. Turnover is introduced by stochastically removing cross-linkers, motors, and filaments from the network with rates $1 / \tau_{c}$, $1 / \tau_{m}$ and $1 / \tau_{f}$. For simplicity, turnover rates of crosslinkers are taken here independent of the forces they sustain. Note that the load dependency of cross-linking has been shown to influence network clustering [13]. Filaments, motors, and passive cross-linkers are added in random positions in the network with on rates $k_{\mathrm{on}}^{f}, k_{\mathrm{on}}^{m}$, and $k_{\mathrm{on}}^{c}$.

We evaluate the components of the stress tensor $\sigma_{i j}$ $(i, j=x$ or $y)$ acting on the boundary of the box [Fig. 1(a)]. The total stress is given by $\sigma_{i j}=\sigma_{i j}^{f}+\sigma_{i j}^{m}$, with $\sigma_{i j}^{f}$ obtained by summing forces acting within the filaments and $\sigma_{i j}^{m}$ from forces acting within motor rods crossing the boundary of the box (Fig. 1). In a linear elastic or viscous material, $\left\langle\sigma_{i j}^{f}\right\rangle=0$ in the absence of large-scale deformation and where $\langle\cdot\rangle$ denotes averaging over positions in the box [30]. In a nonlinearly elastic material, however, $\left\langle\sigma_{i j}^{f}\right\rangle \neq 0$ [30]. At steady state, the average stress acting within the motors is given by (see Ref. [31] and the Supplemental Material [30])

$$
\left\langle\sigma_{i j}^{m}\right\rangle=c\left\langle d n_{i} n_{j}\right\rangle,
$$

where $\mathbf{n}, d$, and $c$ denote the orientation, the motor-induced force dipole strength, and the concentration of bound motors. When the dipoles are isotropically oriented, $\left\langle\sigma_{i j}^{m}\right\rangle=\sigma^{m} \delta_{i j}$, with $\sigma^{m}=c\langle d\rangle / 2$.

We first performed simulations without a turnover of filaments. We focus on the isotropic component of the stress $\sigma \equiv\left(\sigma_{x x}+\sigma_{y y}\right) / 2$. Without cross-linker turnover, the system reaches a stationary state where the stress $\sigma$ fluctuates around a finite positive value [Fig. 3(a)]. For a small number of cross-linkers $\left(N_{c} \rightarrow 0\right)$, no stress is generated, $\sigma=0$, as motors are taken here to be identical. For nonidentical motors, a small isotropic stress can also be generated in the absence of cross-linkers [30]. Above a critical value of the number of cross-linkers $N_{c}>N_{c}^{*} \simeq N_{f}$, a transition occurs and a positive contractile stress appears in the system [Fig. 3(b)]. When $0.6 N_{f} \lesssim N_{c}<N_{c}^{*}$, filaments aggregate in clusters and hence $\sigma \sim 0$, suggesting that the transition is associated with the network connectivity (see movies M1 and M2 in the Supplemental Material [30]). Such a transition to contractility as a function of the number of cross-linkers has been reported in in vitro reconstituted networks
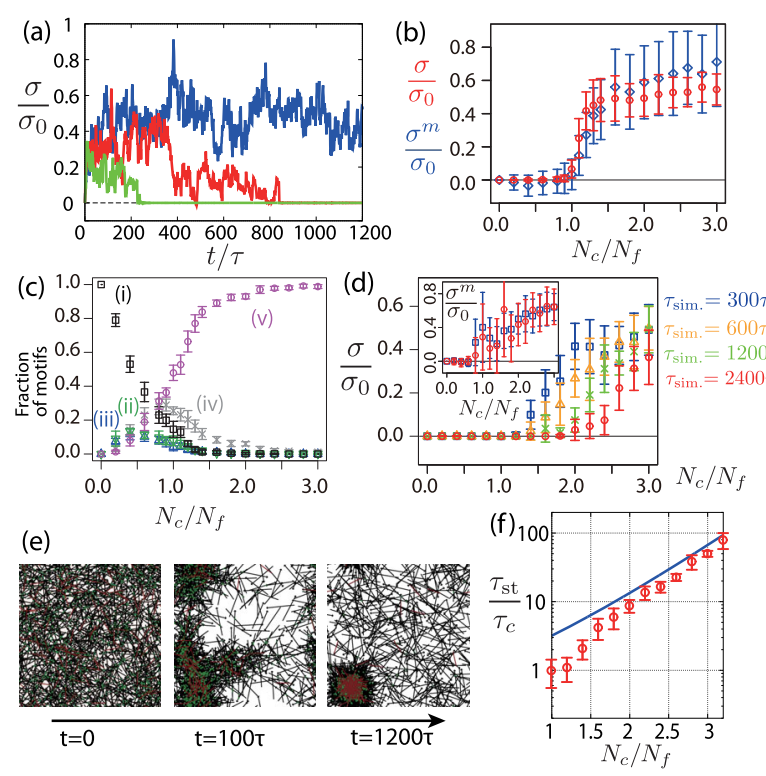

FIG. 3. Stress in a network of motors and filaments, with and without cross-linker turnover. (a) Time evolution of the normalized stress $\sigma / \sigma_{0}$ for $\tau_{c}^{-1}=0, N_{c}=1.2 N_{f}$ (blue), $\tau_{c}=100 \tau$, $N_{c}=1.6 N_{f}$ (red), and $\tau_{c}=100 \tau, N_{c}=1.2 N_{f}$ (green) $\left(\sigma_{0}=\right.$ $f_{0} l_{m} N_{m} / W^{2}$ ). (b) Steady-state isotropic stress $\sigma$ (circles) and motor stress $\sigma^{m}$ (diamonds) as a function of the cross-linkers number $N_{c}$ for $\tau_{c}^{-1}=0$. (c) Fraction of the different configurations in Figs. 2(i) $-2(\mathrm{v})$ at steady state as a function of the crosslinker number $N_{c}$ for $\tau_{c}^{-1}=0$. (d) Isotropic stress $\sigma$ as a function of the cross-linker number $N_{c}$, for a finite cross-linker lifetime $\tau_{c}=100 \tau$, and for several simulation times $\left(\tau_{\mathrm{sim}}=\right.$ $300 \tau, 600 \tau, 1200 \tau$, and $2400 \tau)$. Stress within the motors $\sigma_{m}$ is shown in the inset for $\tau_{\text {sim }}=300 \tau$ and $2400 \tau$. (e) Snapshots of a simulated network with cross-linker turnover $\left(N_{c}=1.2 N_{f}\right.$, $\left.\tau_{c}=100 \tau\right)$. (f) Stress decay time $\tau_{\mathrm{st}}$ as a function of the cross-linker number $N_{c}$ with cross-linker turnover $\left(\tau_{c}=100 \tau\right)$. Solid line, theoretical prediction (see the main text). Other parameters are $N_{f}=1000, N_{m}=100, \tau_{f}^{-1}=0, k_{\mathrm{on}}^{c} \tau_{c}=20$, $l_{f}=2 l_{m}, W=10 l_{m}, \tau_{m}=100 \tau$, and $k_{\mathrm{on}}^{m} \tau=20$.

[10,34], as well as network clustering [35]. The average stress within the motors $\sigma^{m}$ is plotted in Fig. 3(b). The stress $\sigma^{m}$ is larger than the total stress for a large number of cross-linker $N_{c}>N_{f}$, implying that the filament network is under compression $\left(\sigma^{f}<0\right)$. For large $N_{c}$, the configurations shown in Fig. 3(c)(v) dominate in the network. The saturating value of $\sigma^{m} / \sigma_{0} \sim 0.6$ is, nevertheless, smaller than the average force dipole obtained by averaging all possible filament orientations $\langle d\rangle /\left(2 f_{0} l_{m}\right) \sim 1.0$ [Fig. 2(c)]; this is because some configurations do not relax to equilibrium on the time scale of myosin turnover.

When cross-linkers are allowed to turn over, the average stress first reaches a positive value before decaying to zero [Fig. 3(a), red and green curves], even though the stress within the motors $\sigma^{m}$ is still nonzero [Fig. 3(d)]. The decay of the stress correlates with the collapse of the network in an isolated cluster, where filaments accumulate 
[see Fig. 3(e) and movie M3 in the Supplemental Material [30]]. To evaluate the decay time scale $\tau_{\text {st }}$, we fitted an exponentially decreasing function $\sigma(t)=A \exp \left(-t / \tau_{\mathrm{st}}\right)$ to the simulation results. $\tau_{\mathrm{st}}$ increases exponentially with $N_{c}$ for large $N_{c}$ [Fig. 3(f)]. The time scale $\tau_{\text {st }}$ corresponds to the relaxation Maxwell time on which the network becomes fluid due to turnover of passive cross-linkers enabling network rearrangements. With this picture, we estimate

$$
\tau_{\mathrm{st}} \simeq \frac{\tau_{c}}{n_{c}^{*}}\left(e^{n_{c}^{*}}-1\right)
$$

with $n_{c}^{*}=2 N_{c} / N_{f}$, which accounts for $\tau_{\text {st }}$ for large values of $N_{c} / N_{f}$ [Fig. 3(f)]. To obtain Eq. (3), we assume that filaments with at least one cross-linker are fixed, and only filaments with no cross-linker attachment can rearrange. We then compute the first passage time at which a filament is free from cross-linkers, starting from a configuration where the filament is attached with only one cross-linker [30]. Thus, networks with cross-linker turnover can sustain only a transient stress, on a time scale that increases with cross-linker concentration [36].

We now turn to simulations where both filaments and cross-linkers turn over. Remarkably, the network then evolves towards a steady state with a nonzero positive stress [Figs. 4(a) and 4(b)]. Introducing filament turnover prevents the clustering mechanism observed with crosslinker turnover (see movies M4 and M5 in the Supplemental Material [30]). As in the previous case, a transition from a noncontractile to a contractile network appears when the number of cross-linkers $N_{c}$ is increased [Fig. 4(c)]. The stress $\sigma$ deviates again from the average stress within the motors $\sigma^{m}$ [Fig. 4(c), inset]. The dependency of the fraction of configurations as a function of $N_{c}$ is qualitatively similar to the case without filament turnover [Fig. 4(d)].

We then varied the cross-linker number $N_{c}$ and filament turnover time scale $\tau_{f}$ to obtain a phase diagram of stress generation [Fig. 4(e)]. The stress reaches a maximum for intermediate values of the filament turnover time scale $\tau_{f} \sim \tau_{\text {st }}$ [broken line in Fig. 4(e), and Fig. 4(f)]: for slow filament turnover $\tau_{f} \gg \tau_{\mathrm{st}}$, the network collapses in clusters due to cross-linker turnover, while for fast filament turnover $\tau_{f} \ll \tau_{\text {st }}$, filaments are removed before motors reach a configuration where they can exert a force dipole in the network.

An optimal ratio of filament and cross-linker turnover times is therefore needed for contractile stress generation. Interestingly, experimentally measured values of turnover of actin and cross-linker times in the cell cortex, $\tau_{f} \sim$ 15-45 s and $\tau_{c} \sim 7-14 \mathrm{~s}$ [1] yield a ratio of turnover time scale $\tau_{c} / \tau_{f} \sim 0.15-1$, which lies in the region of optimum stress generation near the transition cross-linker density in Fig. 4(f). It will be interesting to investigate stress generation in in vitro experiments where cross-linkers and
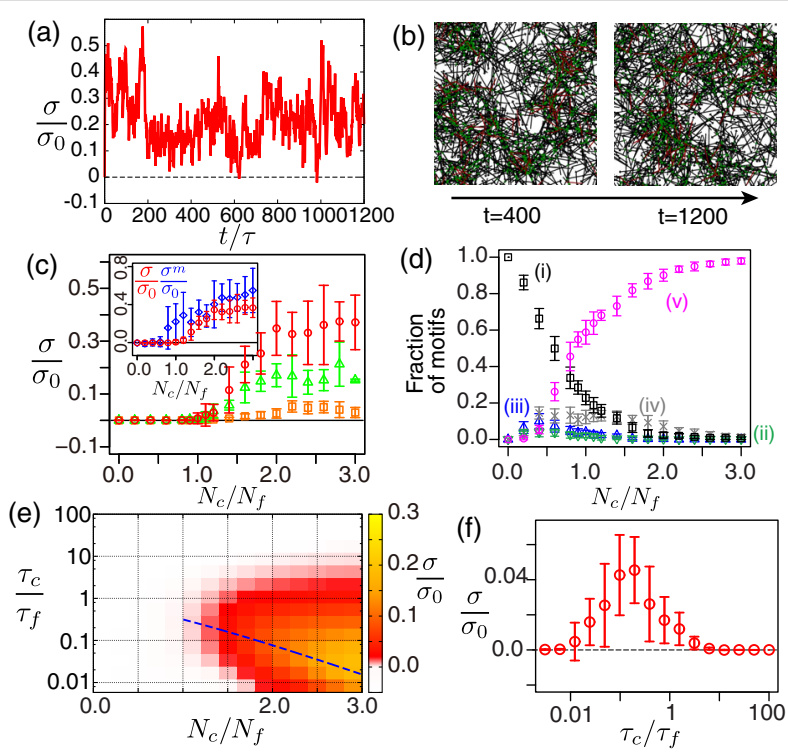

FIG. 4. Stress in a network with filament turnover. (a) Time evolution of the stress in a network with cross-linkers and filament turnover for $\tau_{f}=100 \tau, \tau_{c}=100 \tau$ and $N_{c} / N_{f}=1.6$. (b) Snapshots of the network evolution corresponding to (a). (c) Steady-state stress $\sigma$ as a function of the number of crosslinkers for $\tau_{c}=\tau_{f}=100 \tau$ (circles), $\tau_{c}=\tau_{f}=10 \tau$ (triangles), and $\tau_{c}=\tau_{f}=\tau$ (squares). (Inset) Steady-state stress $\sigma$ (circles) and motor stress $\sigma^{m}$ (diamonds) for $\tau_{c}=\tau_{f}=100 \tau$. (d) Fraction of different configurations (i)-(v) at steady state, as a function of $N_{c}\left(\tau_{c}=100 \tau, \tau_{f}=100 \tau\right)$. (e) Heat map for contractile stress $\sigma$ as a function of $N_{c}$ and $\tau_{c} / \tau_{f}\left(\tau_{c}=\tau\right)$. The broken line indicates $\tau_{f}=\tau_{\mathrm{st}}$. (f) Stress as a function of the ratio of filament and crosslinker turnover rate $\left(\tau_{c}=\tau, N_{c}=1.6 N_{f}\right)$. Other parameters are as in Fig. 3, except that $\tau_{m}^{-1}=0$ and $k_{\mathrm{on}}^{f} \tau_{f}=20$.

filaments are allowed to turn over [37]. Future work will also have to investigate the role of motor multivalency, and cross-linker and motor angular elasticity in stress generation.

We thank Matthew Smith for the helpful discussions. This work was supported partly by the JSPS Institutional Program for Young Researcher Overseas Visits (T. H.), the Postdoctoral Research Fellowship of the Alexander von Humboldt foundation (T.H.), the JSPS Core-to-Core Program (T. H.), the Max Planck Gesellschaft (G. S.), and the Francis Crick Institute which receives its core funding from Cancer Research UK, the UK Medical Research Council, and the Wellcome Trust (G. S).

[1] G. Salbreux, G. Charras, and E. Paluch, Actin cortex mechanics and cellular morphogenesis, Trends Cell Biol. 22, 536 (2012).

[2] J.-F. Joanny and J. Prost, Active gels as a description of the actin-myosin cytoskeleton, HFSP J. 3, 94 (2009).

[3] M. Mayer, M. Depken, J. S. Bois, F. Jülicher, and S. W. Grill, Anisotropies in cortical tension reveal the physical 
basis of polarizing cortical flows, Nature (London) 467, 617 (2010).

[4] M. Behrndt, G. Salbreux, P. Campinho, R. Hauschild, F. Oswald, J. Roensch, S. W. Grill, and C.-P. Heisenberg, Forces driving epithelial spreading in zebrafish gastrulation, Science 338, 257 (2012).

[5] M. Murrell and M. L. Gardel, Actomyosin sliding is attenuated in contractile biomimetic cortices, Mol. Biol. Cell 25, 1845 (2014).

[6] K. Kruse and F Jülicher, Actively Contracting Bundles of Polar Filaments, Phys. Rev. Lett. 85, 1778 (2000).

[7] T. B. Liverpool, M. C. Marchetti, J.-F. Joanny, and J. Prost, Mechanical response of active gels, Europhys. Lett. 85, 18007 (2009).

[8] M. S. e Silva, M. Depken, B. Stuhrmann, M. Korsten, F. C. MacKintosh, and G.H. Koenderink, Active multistage coarsening of actin networks driven by myosin motors, Proc. Natl. Acad. Sci. U.S.A. 108, 9408 (2011).

[9] N. L. Dasanayake, P. J. Michalski, and A. E. Carlsson, General Mechanism of Actomyosin Contractility, Phys. Rev. Lett. 107, 118101 (2011).

[10] S. Köhler and A. R. Bausch, Contraction mechanisms in composite active actin networks, PLoS One 7, e39869 (2012).

[11] M. Lenz, M. L. Gardel, and A. R. Dinner, Requirements for contractility in disordered cytoskeletal bundles, New J. Phys. 14, 033037 (2012).

[12] M. Lenz, T. Thoresen, M. L. Gardel, and A. R. Dinner, Contractile Units in Disordered Actomyosin Bundles Arise from F-Actin Buckling, Phys. Rev. Lett. 108, 238107 (2012).

[13] J. Alvarado, M. Sheinman, A. Sharma, F. C. MacKintosh, and G. H. Koenderink, Molecular motors robustly drive active gels to a critically connected state, Nat. Phys. 9, 591 (2013).

[14] M. Lenz, Geometrical Origins of Contractility in Disordered Actomyosin Networks, Phys. Rev. X 4, 041002 (2014).

[15] D. B. Oelz, B. Y. Rubinstein, and A. Mogilner, A combination of actin treadmilling and cross-linking drives contraction of random actomyosin arrays, Biophys. J. 109, 1818 (2015).

[16] M. Guha, M. Zhou, and Y.-L. Wang, Cortical actin turnover during cytokinesis requires myosin II, Curr. Biol. 15, 732 (2005).

[17] J.-Y. Tinevez, U. Schulze, G. Salbreux, J. Roensch, J.-F. Joanny, and E. Paluch, Role of cortical tension in bleb growth, Proc. Natl. Acad. Sci. U.S.A. 106, 18581 (2009).

[18] K. Tawada and K. Sekimoto, A physical model of ATPinduced actin-myosin movement in vitro, Biophys. J. 59, 343 (1991).

[19] Y. Imafuku, Y. Emoto, and K. Tawada, A protein friction model of the actin sliding movement generated by myosin in mixtures of MgATP and MgGTP in vitro, J. Theor. Biol. 199, 359 (1999).

[20] F. Jülicher and J. Prost, Cooperative Molecular Motors, Phys. Rev. Lett. 75, 2618 (1995).

[21] N. Morone, T. Fujiwara, K. Murase, R. S. Kasai, H. Ike, S. Yuasa, J. Usukura, and A. Kusumi, Three-dimensional reconstruction of the membrane skeleton at the plasma membrane interface by electron tomography, J. Cell Biol. 174, 851 (2006).

[22] G. T. Charras, C.-K. Hu, M. Coughlin, and T. J. Mitchison, Reassembly of contractile actin cortex in cell blebs, J. Cell Biol. 175, 477 (2006).

[23] A. Ott, M. Magnasco, A. Simon, and A. Libchaber, Measurement of the persistence length of polymerized actin using fluorescence microscopy, Phys. Rev. E 48, R1642 (1993).

[24] R. Ishikawa, T. Sakamoto, T. Ando, S. Higashi-Fujime, and K. Kohama, Polarized actin bundles formed by human fascin-1: Their sliding and disassembly on myosin II and myosin V in vitro, J. Neurochem. 87, 676 (2003).

[25] E. W. Kubalek, T. Q. Uyeda, and J. A. Spudich, A dictyostelium myosin II lacking a proximal 58-kDa portion of the tail is functional in vitro and in vivo, Mol. Biol. Cell 3, 1455 (1992).

[26] G. K. Batchelor, Slender-body theory for particles of arbitrary cross-section in Stokes flow, J. Fluid Mech. 44, 419 (1970).

[27] D. Richter, K. Binder, B. Ewen, and B. Stuehn, Screening of hydrodynamic interactions in dense polymer solutions: A phenomenological theory and neutron-scattering investigations, J. Phys. Chem. 88, 6618 (1984).

[28] S. Mukhina, Y.-L. Wang, and M. Murata-Hori, Alphaactinin is required for tightly regulated remodeling of the actin cortical network during cytokinesis, Dev. Cell 13, 554 (2007).

[29] E. M. Reichl, Y. Ren, M. K. Morphew, M. Delannoy, J. C. Effler, K. D. Girard, S. Divi, P. A. Iglesias, S. C. Kuo, and D. N. Robinson, Interactions between myosin and actin crosslinkers control cytokinesis contractility dynamics and mechanics, Curr. Biol. 18, 471 (2008).

[30] See Supplemental Material at http://link.aps.org/ supplemental/10.1103/PhysRevLett.116.188101, which includes Refs. [9,11,31-33], for details of derivations and additional simulation results.

[31] R. A. Simha and S. Ramaswamy, Hydrodynamic Fluctuations and Instabilities in Ordered Suspensions of SelfPropelled Particles, Phys. Rev. Lett. 89, 058101 (2002).

[32] C. W. Gardiner, Stochastic Methods (Springer-Verlag, Berlin, 1985).

[33] P. A. Pury and M. O. Cáceres, Mean first-passage and residence times of random walks on asymmetric disordered chains, J. Phys. A 36, 2695, 2003.

[34] P. M. Bendix, G. H. Koenderink, D. Cuvelier, Z. Dogic, B. N. Koeleman, W. M. Brieher, C. M. Field, L. Mahadevan, and D. A. Weitz, A quantitative analysis of contractility in active cytoskeletal protein networks, Biophys. J. 94, 3126 (2008).

[35] S. Köhler, V. Schaller, and A. R. Bausch, Structure formation in active networks, Nat. Mater. 10, 462 (2011).

[36] W. Jung, M. P. Murrell, and T. Kim, F-actin cross-linking enhances the stability of force generation in disordered actomyosin networks, Comput. Part. Mech. 2, 317 (2015).

[37] E. A. Shah and K. Keren, Symmetry breaking in reconstituted actin cortices, eLife 3, e01433 (2014). 